\title{
8-羊基喹啉-5-磺酸构筑的有机-无机配位化合物的 合成、结构和性质研究
}

\author{
王瑛，薛铭，徐家宁，朱广山，装式纶* \\ 吉林大学无机合成与制备国家重点实验室, 长春 130012 \\ * 通讯作者, E-mail: sqiu@mail.jlu.edu.cn
}

收稿日期：2009-07-16；接受日期：2009-08-10

摘要在溶剂热条件下, 以 8-差基喹啉-5-磺酸为配体, 合成了两个新的有机-无机 配位化合物 $\mathrm{Mn}(\mathrm{QS})\left(\mathrm{H}_{2} \mathrm{O}\right)(\mathbf{1})$ 和 $\mathrm{Co}(\mathrm{QS})\left(\mathrm{H}_{2} \mathrm{O}\right)_{2}$ (2) ( $\mathrm{H}_{2} \mathrm{QS}=8$-hydroxylquinoline-5sulfonic acid), 并且对他们进行了单晶X-射线解析. 其中化合物 $\mathbf{1}$ 是一个具有金红石 拓扑的三维开放骨架结构, 化合物 2 是一个三维超分子结构. 我们对这些化合物进 行了粉末X射线, 红外光谱, 差热热重, 荧光光谱和磁学性质的研究.

\section{关键词}

有机-无机杂化材料

8-差基喹啉-5-磺酸

荧光

磁性

\section{1 引言}

近年来, 有机-无机杂化材料由于其有趣的网络 拓扑结构和作为功能材料有着潜在的应用前景，而 受到了人们的广泛关注 ${ }^{[1 \sim 6]}$. 拓扑是指用简单的节点 和连接来代替复杂的多维结构, 它能够有效地分析、 设计和比较网络结构 ${ }^{[7-12]}$. 人们通过用多原子的有机 配体来代替单原子的阴离子作为连接, 以几何上匹 配的分子基团代替矿物拓扑结构中的节点, 已经成 功地合成出钙钛矿、石英、金刚石、PtS、长石和金 红石等很多矿石结构 ${ }^{[13 ~ 18]}$.

目前，寻找有效的路线来合成具有预期应用前 景和有趣结构的有机-无机聚合物仍然是一个富有挑 战性的研究工作. 为了得到新颖的多功能的有机-无 机杂化材料，人们应用了各种无机金属中心和有机 配体分子. 众所周知, 含有芳香羧酸基团的有机配体 因为能够和大部分的金属离子配位，所以一直是人 们关注的焦点. 磺酸基由于被认为是不容易取代溶 剂分子而与金属离子配位的基团 ${ }^{[19,20]}$, 因而含有磺 酸基的有机配体被人们所忽视，还没有被人们系统
的研究. 为了得到新颖的有机-无机配位聚合物, 我 们选择了相对研究较少的 8-差基喹啉-5-磺酸 $\left(\mathrm{H}_{2} \mathrm{QS}\right)$ 作为有机配体 ${ }^{[21 ~ 23]}$, 过渡金属锰和钴作为金属中心, 在溶剂热合成条件下，得到了一个具有金红石拓扑 结构的三维化合物 $\mathrm{Mn}(\mathrm{QS})\left(\mathrm{H}_{2} \mathrm{O}\right)(\mathbf{1})$ 和一个具有三维 超分子结构的化合物 $\mathrm{Co}(\mathrm{QS})\left(\mathrm{H}_{2} \mathrm{O}\right)_{2}$ (2). 这里我们对 这两个化合物的合成、晶体结构、苂光前景和磁学性 质进行了研究.

\section{2 实验部分}

\section{1 试剂和仪器}

$\mathrm{MnCl}_{2} \cdot 4 \mathrm{H}_{2} \mathrm{O}\left(\mathrm{AR}\right.$, 北京化工厂), $\mathrm{Co}\left(\mathrm{NO}_{3}\right)_{2} \cdot 6 \mathrm{H}_{2} \mathrm{O}$ (AR, 北京化工厂)，8-羊基喹啉-5-磺酸 $\left(\mathrm{H}_{2} \mathrm{QS}, \mathrm{AR}\right.$, Aldrich), 环己醇(AR, 天津市化学试剂二厂), $N, N$-二 甲基甲酰胺(DMF, AR, 天津天泰精细化学品有限公 司), 咪唑(AR, 北京化工厂), 水为蒸馏水. 元素分析 在Perkin-Elmer 2004 元素分析仪上测定; 晶体数据在 Bruke SMART CCD上收集; 红外光谱在Nicolet Impact 410 FTIR红外光谱仪上测试; 苂光光谱在LS 55 荧光/磷光光谱仪上测试, 变温磁化率测定采用 
Quantum Design Model MPMS SQUID磁力计测量的.

\section{2 化合物 $\mathrm{Mn}(\mathrm{QS})\left(\mathrm{H}_{2} \mathrm{O}\right)(1)$ 的合成}

将反应物 $\mathrm{MnCl}_{2} \cdot 4 \mathrm{H}_{2} \mathrm{O}(0.040 \mathrm{~g}, 0.2 \mathrm{mmol})$ 和 $\mathrm{H}_{2} \mathrm{QS}(0.024 \mathrm{~g}, 0.1 \mathrm{mmol})$ 加入到 $6 \mathrm{~mL} \mathrm{H} \mathrm{H}_{2} \mathrm{O}$ 和 $2 \mathrm{~mL}$ DMF 的混合溶液中, 在室温下摚拌, 然后向此混合 溶液中加入 $0.005 \mathrm{~g}$ 咪唑继续搅拌大约 $0.5 \mathrm{~h}$, 将混合 溶液 装入 $15 \mathrm{~mL}$ 聚四氟乙烯内衬的不锈钢反应釜中, 在自生压力的条件下, $140^{\circ} \mathrm{C}$ 烘箱中放置 5 天, 自然冷 却至室温, 将晶体过滤用无水乙醇洗涤, 得到黄色块 状晶体. 元素分析理论值(实验值, \%): C 36.47 (36.69), H 2.36 (2.58), N 4.72 (4.64).

\section{3 化合物 $\mathrm{Co}(\mathrm{QS})\left(\mathrm{H}_{2} \mathrm{O}\right)_{2}(2)$ 的合成}

将反应物 $\mathrm{Co}\left(\mathrm{NO}_{3}\right)_{2} \cdot 6 \mathrm{H}_{2} \mathrm{O}(0.029 \mathrm{~g}, 0.1 \mathrm{mmol})$ 和 $\mathrm{H}_{2} \mathrm{QS}(0.048 \mathrm{~g}, 0.2 \mathrm{mmol})$ 加入到 $6 \mathrm{~mL} \mathrm{H}_{2} \mathrm{O}$ 和 $2 \mathrm{~mL}$ 环己醇的混合溶液中, 在室温下搅拌, 用 $2 \mathrm{~mol} / \mathrm{L}$ 的 $\mathrm{NaOH}$ 溶液调节 $\mathrm{pH}$ 值 6 7, 然后将此混合溶液装入到 $15 \mathrm{~mL}$ 聚四氟乙烯内衬的不锈钢反应釜中，在自生压 力的条件下， $160^{\circ} \mathrm{C}$ 烘箱中放置 10 天，自然冷却至室 温, 将晶体过滤用无水乙醇洗涤, 得到红色块状晶体. 元素分析理论值(实验值, \%): C 33.95 (33.86), H 2.83 (2.89), N 4.40 (4.42).

\section{4 晶体结构的测定}

在 $293 \mathrm{~K}$ 下，分别选取尺寸大小合适的黄色和红 色块状晶体, 在Bruke SMART X-射线单晶衍射仪上, 以石墨单色化的Mo $K \alpha$ 射线 $(\lambda=0.071073 \mathrm{~nm})$ 为衍射 源，采用 $\omega-2 \theta$ 扫描方式收集衍射点，吸收校正用程 序SADABS完成 ${ }^{[24]}$. 以可观测的独立衍射点进行结 构计算. 晶体结构由直接法解出, 非氢原子坐标在以 后的逐次差值Fourier合成中逐步确定，对全部非氢 原子坐标及各向异性参数进行全矩阵最小二乘方法 修正. 所有计算均用SHELXL-97 程序完成 ${ }^{[25]}$. 化合 物 1 和 2 的晶体学参数总结在表 1 中, 选择的键长和 键角在表 2 中给出.

\section{3 结果与讨论}

\section{1 化合物 1 的晶体结构}

单晶X射线分析表明化合物 1 为单斜晶系, $P 2_{1} / c$ 空间群. 在化合物 $\mathbf{1}$ 的不对称结构单元中包含有 17
表 1 化合物 1 和 2 的晶体学参数 ${ }^{a)}$

\begin{tabular}{|c|c|c|}
\hline Compound & 1 & 2 \\
\hline Empirical formula & $\mathrm{C}_{9} \mathrm{H}_{7} \mathrm{MnNO}_{5} \mathrm{~S}$ & $\mathrm{C}_{9} \mathrm{H}_{9} \mathrm{CoNO}_{6} \mathrm{~S}$ \\
\hline Formula weight & 296.16 & 318.16 \\
\hline Temperature & 293(2) K & 293(2) K \\
\hline Wavelength & $0.071073 \mathrm{~nm}$ & $0.071073 \mathrm{~nm}$ \\
\hline Crystal system & Monoclinic & Orthorhombic \\
\hline Space group & $P 2_{1} / c$ & $P b c a$ \\
\hline$a(\mathrm{~nm})$ & $0.82903(12)$ & $0.92808(3)$ \\
\hline$b(\mathrm{~nm})$ & $1.32778(19)$ & $1.52263(6)$ \\
\hline$c(\mathrm{~nm})$ & $0.91327(14)$ & $1.61552(6)$ \\
\hline$\beta\left({ }^{\circ}\right)$ & $99.633(2)$ & 90 \\
\hline$V\left(\mathrm{~nm}^{3}\right) \times 10^{3}$ & $991.1(3)$ & $2282.93(14)$ \\
\hline$Z$ & 4 & 8 \\
\hline$D_{\mathrm{c}}\left(\mathrm{g} \cdot \mathrm{cm}^{-3}\right)$ & 1.985 & 1.851 \\
\hline$\mu\left(\mathrm{mm}^{-1}\right)$ & 1.551 & 1.705 \\
\hline$F(000)$ & 596 & 1288 \\
\hline$\theta_{\text {range }}\left({ }^{\circ}\right)$ & $2.49 \sim 28.28$ & $2.52 \sim 28.26$ \\
\hline $\begin{array}{l}\text { Reflections collected/ } \\
\text { unique }\end{array}$ & $\begin{array}{l}6188 / 2269 \\
{[R(\text { int })=0.0331]}\end{array}$ & $\begin{array}{l}13336 / 2689 \\
{[R(\text { int })=0.0363]}\end{array}$ \\
\hline $\begin{array}{l}\text { Data/ restraints / } \\
\text { parameters }\end{array}$ & $2269 / 0 / 182$ & $2689 / 0 / 199$ \\
\hline Goodness-of-fit on $F^{2}$ & 0.962 & 1.045 \\
\hline Final $R_{\text {indices }}(I>2 o(I))$ & $\begin{array}{l}R_{1}=0.0324 \\
\mathrm{w} R_{2}=0.0753\end{array}$ & $\begin{array}{l}R_{1}=0.0326 \\
\mathrm{w} R_{2}=0.0860\end{array}$ \\
\hline$R_{\text {indices (all data) }}$ & $\begin{array}{l}R_{1}=0.0418 \\
\mathrm{w} R_{2}=0.0786\end{array}$ & $\begin{array}{l}R_{1}=0.0372, \\
\mathrm{w} R_{2}=0.0888\end{array}$ \\
\hline
\end{tabular}

a) $R_{1}=\Sigma|| F_{\mathrm{O}}|-| F_{\mathrm{c}}|| \Sigma\left|F_{\mathrm{O}}\right|, w R_{2}=\left|\Sigma w\left(\left|F_{\mathrm{O}}\right|^{2}-|F \mathrm{c}|^{2}\right)\right| / \Sigma\left|w\left(F_{\mathrm{O}}\right)^{2}\right|^{1 / 2}$

个非氢原子, 1 个 $\mathrm{Mn}^{2+}, 9$ 个 $\mathrm{C}$ 原子, 1 个 $\mathrm{N}$ 原子, 5 个 $\mathrm{O}$ 原子和 1 个 $\mathrm{S}$ 原子. 如图 1 所示 $\mathrm{Mn}$ 金属中心采取八面 体配位几何构型，与 5 个氧原子 $(\mathrm{O} 1 \mathrm{w}, \mathrm{O} 2, \mathrm{O} 2 \mathrm{~A}, \mathrm{O} 3$, $\mathrm{O} 5 \mathrm{~A})$ 和 1 个氮原子 $(\mathrm{N} 1)$ 配位, $\mathrm{O} 1 \mathrm{w}$ 来自于配位的端基 水分子, $\mathrm{O} 2, \mathrm{O} 2 \mathrm{~A}$ 来自于配体 $\mathrm{QS}$ 的羟基, $\mathrm{O} 3, \mathrm{O} 5 \mathrm{~A}$ 来于 配体 $\mathrm{QS}$ 的磺酸基. $\mathrm{Mn}-\mathrm{O}$ 键的键长有三种分别是 $\mathrm{Mn}$ $-\mathrm{O} 1 \mathrm{w}$ 的键长为 $0.2178 \mathrm{~nm}, \mathrm{Mn}-\mathrm{O} 2$ 的键长为 0.2126 $\mathrm{nm}, \mathrm{Mn}-\mathrm{O} 3$ 的键长为 $0.2200 \mathrm{~nm}, \mathrm{Mn}-\mathrm{N}$ 的键长为 $0.2279 \mathrm{~nm}, \mathrm{O}-\mathrm{Mn}-\mathrm{O}$ 键角从 $74.40^{\circ}$ 到 $171.11^{\circ}, \mathrm{O}-$ $\mathrm{Mn}-\mathrm{N}$ 键角从 $73.57^{\circ}$ 到 $147.97^{\circ}$, 这些键长和键角与 文献中所报道的这类材料的键长和键角相近 ${ }^{[26]}$. 从 以上的分析我们可以得出 $\mathrm{Mn}$ 金属中心的八面体构型 $\mathrm{MnO}_{5} \mathrm{~N}$ 为扭曲的八面体. 两个晶体学上等价的扭曲 的八面体 $\mathrm{MnO}_{5} \mathrm{~N}$ 通过两个 $\mathrm{Q} \mathrm{S}$ 上的羟基 
表 2 化合物 1 和 2 的选择的键长 $(\mathrm{nm})$ 和键角 $\left(^{(}\right)$表

\begin{tabular}{|c|c|c|c|}
\hline \multicolumn{4}{|c|}{ 化合物 1} \\
\hline $\mathrm{Mn}(1)-\mathrm{O}(2)$ & $0.2126(15)$ & $\mathrm{Mn}(1)-\mathrm{O}(3)$ & $0.2200(17)$ \\
\hline $\left.\operatorname{Mn}(1)-O(2)^{a}\right)$ & $0.2171(15)$ & $\operatorname{Mn}(1)-O(5)^{b)}$ & $0.2204(16)$ \\
\hline $\mathrm{Mn}(1)-\mathrm{O}(1 \mathrm{~W})$ & $0.2178(2)$ & $\mathrm{Mn}(1)-\mathrm{N}(1)$ & $0.2278(19)$ \\
\hline $\mathrm{O}(2)-\mathrm{Mn}(1)-\mathrm{O}(2)^{\mathrm{a})}$ & $74.40(6)$ & $\mathrm{O}(1 \mathrm{~W})-\mathrm{Mn}(1)-\mathrm{O}(5)^{\mathrm{b})}$ & $83.08(9)$ \\
\hline $\mathrm{O}(2)-\mathrm{Mn}(1)-\mathrm{O}(1 \mathrm{~W})$ & $91.78(9)$ & $\mathrm{O}(3)-\mathrm{Mn}(1)-\mathrm{O}(5)^{\mathrm{b})}$ & $81.90(7)$ \\
\hline $\mathrm{O}(2)^{\mathrm{a}}-\mathrm{Mn}(1)-\mathrm{O}(1 \mathrm{~W})$ & $99.00(9)$ & $\mathrm{O}(2)-\mathrm{Mn}(1)-\mathrm{N}(1)$ & $147.97(6)$ \\
\hline $\mathrm{O}(2)-\mathrm{Mn}(1)-\mathrm{O}(3)$ & $92.65(6)$ & $\mathrm{O}(2)^{\mathrm{a})}-\mathrm{Mn}(1)-\mathrm{N}(1)$ & $73.57(6)$ \\
\hline $\mathrm{O}(2)^{\mathrm{a}}-\mathrm{Mn}(1)-\mathrm{O}(3)$ & $96.01(6)$ & $\mathrm{O}(1 \mathrm{~W})-\mathrm{Mn}(1)-\mathrm{N}(1)$ & $93.26(10)$ \\
\hline $\mathrm{O}(1 \mathrm{~W})-\mathrm{Mn}(1)-\mathrm{O}(3)$ & $164.98(9)$ & $\mathrm{O}(3)-\mathrm{Mn}(1)-\mathrm{N}(1)$ & $90.57(7)$ \\
\hline $\mathrm{O}(2)-\mathrm{Mn}(1)-\mathrm{O}(5)^{\mathrm{b})}$ & $107.62(6)$ & $\mathrm{O}(5)^{\mathrm{b})}-\mathrm{Mn}(1)-\mathrm{N}(1)$ & $104.38(7)$ \\
\hline $\mathrm{O}(2)^{\mathrm{a}}-\mathrm{Mn}(1)-\mathrm{O}(5)^{\mathrm{b})}$ & $177.11(6)$ & & \\
\hline \multicolumn{4}{|c|}{ 化合物 2} \\
\hline $\mathrm{Co}(1)-\mathrm{O}(5 \mathrm{~W})$ & $0.2039(16)$ & $\operatorname{Co}(1)-\mathrm{N}(1)$ & $0.2110(17)$ \\
\hline $\left.\operatorname{Co}(1)-O(1)^{a}\right)$ & $0.2059(14)$ & $\mathrm{Co}(1)-\mathrm{O}(6 \mathrm{~W})$ & $0.2144(17)$ \\
\hline $\mathrm{Co}(1)-\mathrm{O}(1)$ & $0.2064(13)$ & $\mathrm{Co}(1)-\mathrm{O}(2)$ & $0.2184(14)$ \\
\hline $\mathrm{O}(5 \mathrm{~W})-\mathrm{Co}(1)-\mathrm{O}(1)^{\mathrm{a})}$ & $174.65(7)$ & $\mathrm{O}(1)-\mathrm{Co}(1)-\mathrm{O}(6 \mathrm{~W})$ & $87.87(6)$ \\
\hline $\mathrm{O}(5 \mathrm{~W})-\mathrm{Co}(1)-\mathrm{O}(1)$ & $107.03(7)$ & $\mathrm{N}(1)-\mathrm{Co}(1)-\mathrm{O}(6 \mathrm{~W})$ & $88.77(6)$ \\
\hline $\mathrm{O}(1)^{\mathrm{a})}-\mathrm{Co}(1)-\mathrm{O}(1)$ & $76.65(6)$ & $\mathrm{O}(5 \mathrm{~W})-\mathrm{Co}(1)-\mathrm{O}(2)$ & $81.77(7)$ \\
\hline $\mathrm{O}(5 \mathrm{~W})-\mathrm{Co}(1)-\mathrm{N}(1)$ & $98.10(7)$ & $\mathrm{O}(1)^{\mathrm{a})}-\mathrm{Co}(1)-\mathrm{O}(2)$ & $94.34(6)$ \\
\hline $\mathrm{O}(1)^{\mathrm{a})}-\mathrm{Co}(1)-\mathrm{N}(1)$ & $78.57(6)$ & $\mathrm{O}(1)-\mathrm{Co}(1)-\mathrm{O}(2)$ & $91.69(5)$ \\
\hline $\mathrm{O}(1)-\mathrm{Co}(1)-\mathrm{N}(1)$ & $154.57(6)$ & $\mathrm{N}(1)-\mathrm{Co}(1)-\mathrm{O}(2)$ & $95.80(6)$ \\
\hline $\mathrm{O}(5 \mathrm{~W})-\mathrm{Co}(1)-\mathrm{O}(6 \mathrm{~W})$ & $88.87(7)$ & $\mathrm{O}(6 \mathrm{~W})-\mathrm{Co}(1)-\mathrm{O}(2)$ & $170.07(6)$ \\
\hline $\mathrm{O}(1)^{\mathrm{a})}-\mathrm{Co}(1)-\mathrm{O}(6 \mathrm{~W})$ & $95.21(7)$ & & \\
\hline
\end{tabular}

Symmetry transformations used to generate equivalent atoms: a): $-x,-y+2,-z+2$; b): $x,-y+3 / 2, z+1 / 2$

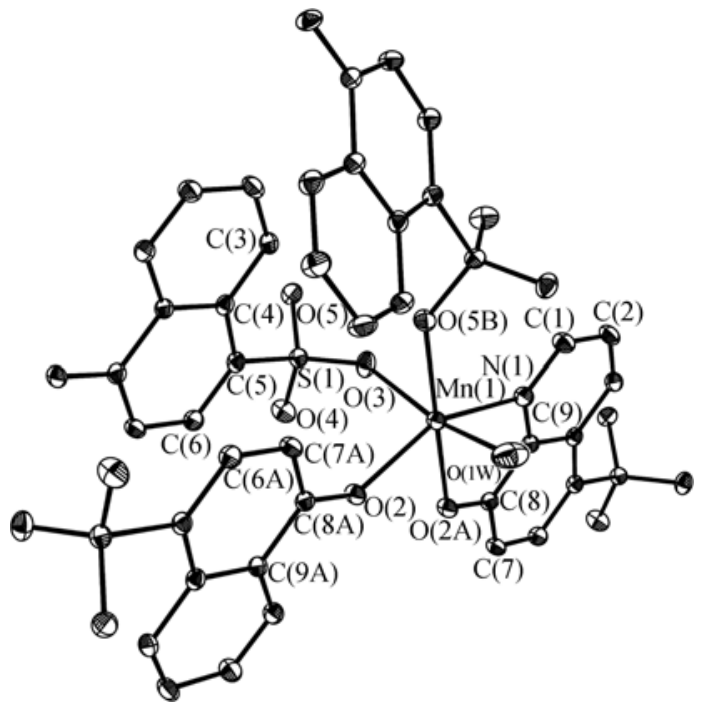

图 1 化合物 1 的配位环境图 省略了氢原子

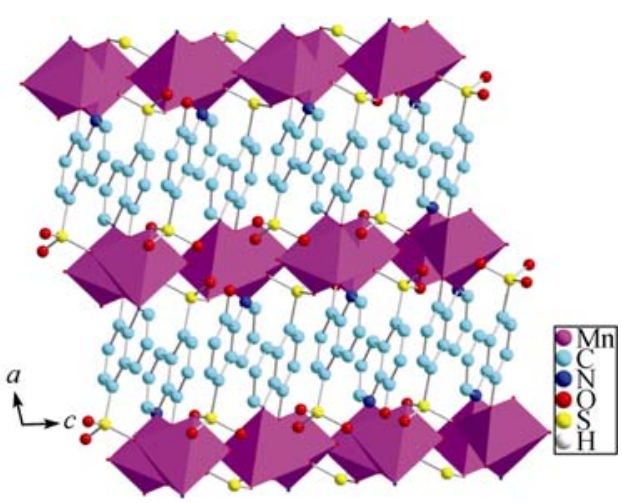

图 2 化合物 1 在(101)平面上的堆积图

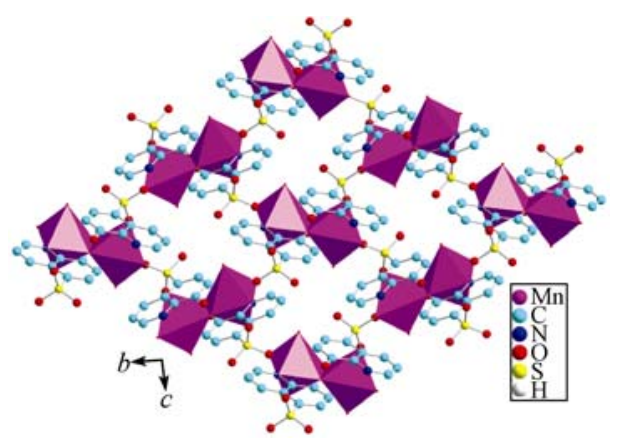

图 3 化合物 1 在(100)平面上的十六元环孔道结构

氧原子桥联形成了一个双金属的次级结构单元，这 些双金属的次级结构单元通过 QS 的磺酸基的两个氧 原子桥联在一起沿 $b$ 轴形成了无限的无机链. 这些无 机链通过 QS 的喹啉环相互连接形成了一个三维骨架 结构如图 2 所示在(101)平面上观察的无机链通过喹 啉环相互连接起来, 在相邻的喹啉环之间存在着较 强的 $\pi-\pi$ 相互作用. 这些无机链和喹啉环在 $(100)$ 平面 上形成了具有十六元环的孔道结构, 如图 3 所示.

为了更好地了解这些化合物的结构和拓扑原型, 我们仔细地分析金属中心与配体的连接方式. 如图 4(a)所示, 两个金属 Mn 形成的双金属的次级结构单 元与六个 $\mathrm{QS}$ 配体相互连接, 因此我们把双金属形成 的次级结构单元看作是一个无机的六连接节点. 同 样的如图 4(b)所示, 每个 QS 配体分别通过磺酸基, 喹啉环上的 $\mathrm{N}$ 和羟基 $\mathrm{O}$ 与四个金属 $\mathrm{Mn}$ 相连，由于与 $\mathrm{N}$ 和羟基 $\mathrm{O}$ 相连的两个金属 $\mathrm{Mn}$ 是通过羟基的 $\mathrm{O}$ 桥 联形成双金属次级结构单元，我们把这两个 $\mathrm{Mn}$ 看作 是一个节点, 这样有机配体 $\mathrm{QS}$ 看作是一个有机的三 


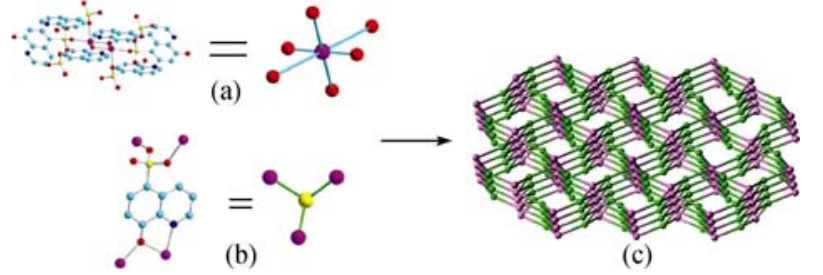

图 4 (a) 两个金属 $\mathrm{Mn}$ 形成的二聚体与刘个 $\mathrm{QS}$ 配体相连, 形成了一个无机的六连接的节点; (b) QS 配体与四个金属 Mn 相连, 形成了一个有机的三连接的节点; (c)无机的六连 接的节点与有机的三连接的节点形成的具有金红石的拓扑 结构

连接节点. 把无机的六连接节点与有机的三连接节 点相互连接, 我们发现得到了一个rutile拓扑结构如 图 4(c), 其Schläfli 符号是 $\left(4 \cdot 6^{2}\right)_{2}\left(4^{2} \cdot 6^{10} \cdot 8^{3}\right)$.

\section{2 化合物 2 的晶体结构}

单晶X射线分析表明化合物 2 为正交晶系, $P b c a$ 空间群. 与我们之前合成的镍的化合物结构类似. 在 化合物 2 的每个不对称结构单元中含有 18 个非氢原 子, 包括 1 个 $\mathrm{Co}^{2+}$ 金属中心, 9 个碳原子, 6 个氧原子, 1 个氮原子和 1 个硫原子. 如图 5 所示为化合物 2 的金 属Co的配位结构图，从图中我们可以发现 Co 是采取 六配位八面体的配位方式，与 5 个氧原子 $(\mathrm{O} 1, \mathrm{O} 2$, $\mathrm{O} 1 \mathrm{~A}, \mathrm{O} 5 \mathrm{w}, \mathrm{O} 6 \mathrm{w})$ 和 1 个氮原子(N1)配位. 五个氧原子 中其中 2 个 $(\mathrm{O} 5 \mathrm{w}, \mathrm{O} 6 \mathrm{w})$ 来自于端基水分子, $\mathrm{O} 1$ 和 $\mathrm{O} 1 \mathrm{~A}$ 来自于配体 $\mathrm{QS}$ 上的羟基氧原子, $\mathrm{O} 2$ 是来自于配 体 $\mathrm{QS}$ 上的磺酸基上的氧原子, $\mathrm{N} 1$ 来自于配体 $\mathrm{QS}$, 其 中 $\mathrm{QS}$ 上的 $\mathrm{N}$ 和羟基氧原子采用的是双齿鳌合的配位 方式与金属中心形成了比较稳定的五元环。其中 $\mathrm{Co}-\mathrm{O}$ 健长是从 $0.2039 \mathrm{~nm}$ 到 $0.2184 \mathrm{~nm}, \mathrm{Co}-\mathrm{N}$ 键长 是 $0.2110 \mathrm{~nm}, \mathrm{O}-\mathrm{Co}-\mathrm{O}$ 键角是从 $74.40(6)^{\circ}$ 到 171.11(6) $)^{\circ}, \mathrm{O}-\mathrm{Co}-\mathrm{N}$ 键角是从 $76.65(6)^{\circ}$ 到 $174.65(7)^{\circ}$, 这些键长和键角与文献中所报道的这类 材料的键长和键角相近 ${ }^{[27]}$. 两个晶体学上等价的扭 曲八面体配位的Co通过两个晶体学上等价的羟基氧 原子 $(\mathrm{O} 1, \mathrm{O} 1 \mathrm{~A})$ 相互桥连形成了双金属的次级结构单 元，这些次级结构单元从四个不同的方向通过四个 QS上的磺酸基相互连接形成了一个无限的层状结构. 在相邻的喹啉环之间存在着比较强的 $\pi-\pi$ 相互作用,
在端基配位水分子和单齿配位的磺酸基的氧原子之 间存在着氢键相互作用. 形成氢键的 $\mathrm{O} \cdots \mathrm{O}$ 之间的 距离为 $0.2679 \sim 0.2844 \mathrm{~nm}, \mathrm{O}-\mathrm{H} \cdots \mathrm{O}$ 的键角为 $163 \sim 175^{\circ}$, 这表明了端基配位水分子和单齿配位的磺 酸基的氧原子之间的氢键作用是比较强的. 从[111] 方向观察，我们可以发现化合物 2 形成了类似于梯子 状的结构(如图 6 所示), 从[100]和[001]方向上观察， 这些无限的链通过氢键和 $\pi-\pi$ 相互作用形成了一个三 维的超分子结构.

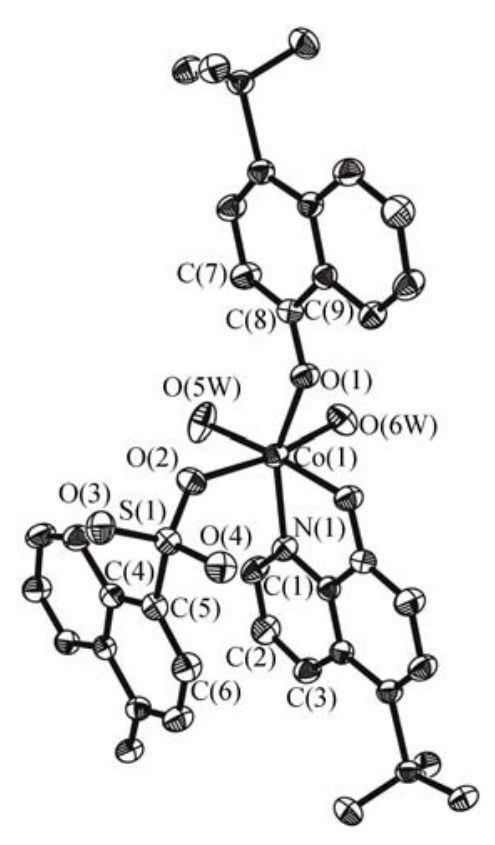

图 5 化合物 2 的配位环境图

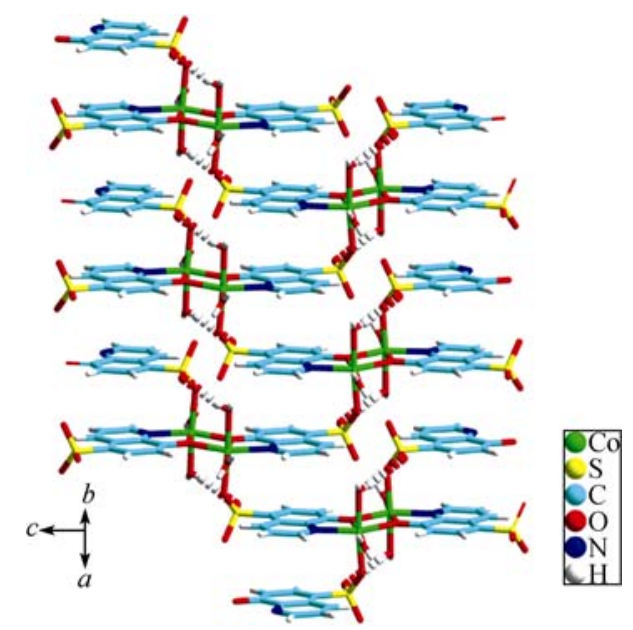

图 6 化合物 2 沿[111]方向的堆积图 
对比这两个化合物的结构，我们可以发现在化 合物 2 中由于两个配位水分子的存在阻止了该化合 物形成三维开放骨架结构, 而在化合物 1 中磺酸基上 的一个 $\mathrm{O}$ 原子取代了配位水分子，使其拓展为三维 结构.

\section{3 红外光谱分析}

化合物 1 和 2 具有相似的红外光谱图. $3437 \mathrm{~cm}^{-1}$ 处的宽峰归属于典型的配位水分子, 在 1650 1392 $\mathrm{cm}^{-1}$ 之间的吸收峰归属于喹啉骨架的 $\mathrm{C}-\mathrm{C}$ 和 $\mathrm{C}-\mathrm{N}$ 键的振动, 在 $1243 \sim 1150 \mathrm{~cm}^{-1}$ 之间的吸收峰归属于 QS配体上的磺酸基一 $\mathrm{SO}_{3}$ 的振动, 在 $837 \sim 619 \mathrm{~cm}^{-1}$ 之间的吸收峰归属于喹啉环上的 $\mathrm{C}-\mathrm{H}$ 键的振动, 在 $650 \mathrm{~cm}^{-1}$ 附近的吸收峰归属于喹啉环上的 $\mathrm{C}-\mathrm{S}$ 键 的振动, 在 $619 \sim 455 \mathrm{~cm}^{-1}$ 之间的吸收峰归属于磺酸 基上的 $\mathrm{S}-\mathrm{O}$ 的伸缩振动 ${ }^{[28,29]}$.

\section{4 热重分析}

为了研究这两个化合物的热稳定性, 我们对它 们进行了热重分析, 加热速率是 $10^{\circ} \mathrm{C} \mathrm{min}^{-1}$. 化合物 1 有两个失重过程, 从室温到 $250^{\circ} \mathrm{C}$ 之前质量没有发 生变化, 晶体保持稳定, 第一个失重发生在 $320^{\circ} \mathrm{C}$ 附 近, 失重大约 $6.05 \%$ 这可以归属为一个配位水分子的 失去 (计算结果为 $6.08 \%$ ), 当温度高于 $420^{\circ} \mathrm{C}$ 时, 化 合物的骨架结构开始分解. 化合物 2 的失重过程也分 为两步，第一个失重发生在 $155 \sim 240^{\circ} \mathrm{C}$ 之间失重约为 $11.71 \%$, 归属于两个配位水分子的失去 (计算值为: $11.31 \%$ ), 在 $240 \sim 400^{\circ} \mathrm{C}$ 之间化合物 2 的结构保持不变, 大于 $400^{\circ} \mathrm{C}$ 之后化合物 $\mathbf{2}$ 的骨架结构发生坞塌.

\section{5 化合物的苂光前景}

在室温下我们研究了化合物 $\mathbf{1}$ 和 $\mathbf{2}$ 的固态苂光光 谱, 如图 7 所示. 对于 8-着基喹啉-5-磺酸，它的激发 波长为 $395 \mathrm{~nm}$, 在 $454 \mathrm{~nm}$ 处有个比较强的发射峰, 这归属于有机配体内部的 $\pi^{*} \rightarrow \pi$ 电荷转移. 对于化 合物 1 和 2 它们在相同的的激发波长 $395 \mathrm{~nm}$ 附近激 发, 最强的发射峰分别为 $568 \mathrm{~nm}$ 和 $575 \mathrm{~nm}$,与有机配 体的发射峰相比大约有 $100 \mathrm{~nm}$ 的红移, 这可以归属 为配体到金属的电荷转移. 化合物 1 和 $\mathbf{2}$ 的荧光性
质的研究可以使得它们在荧光材料方面有着潜在的 应用。

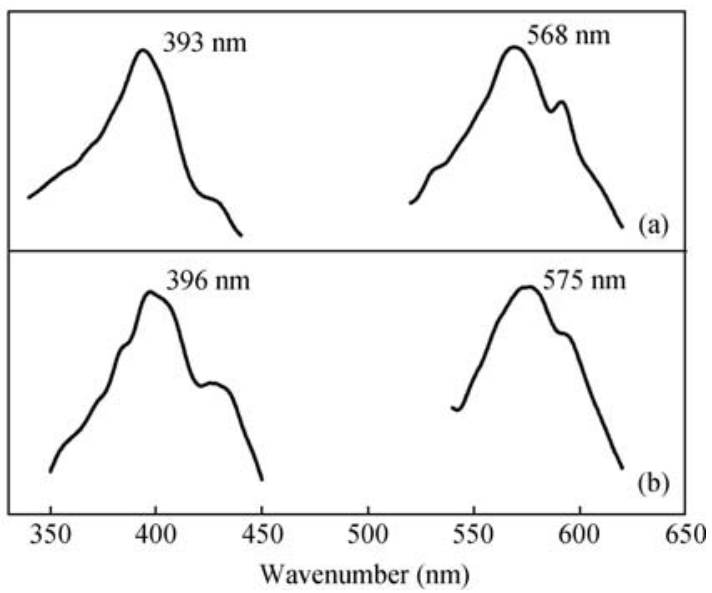

图 7 化合物 1(a)、化合物 2(b)的荧光光谱图

\section{6 磁性研究}

化合物 1 和 2 的变温磁化率测定采用Quantum Design Model MPMS SQUID磁力计在外磁场 5000 Oe 条件下对多晶样品进行从 $4 \mathrm{~K}$ 到 $300 \mathrm{~K}$ 进行测试. 如 图 8(a)所示, 化合物 $\mathbf{1}$ 在 $4 \mathrm{~K}$ 到 $300 \mathrm{~K}$ 范围内的磁化率 按照Curie-Weiss定律 $\left[\chi_{M}=C /(T-\Theta)\right]$ 进行拟合, 得到的 结果是: Curie常数 $C=4.85 \mathrm{~cm}^{3} \cdot \mathrm{K} \cdot \mathrm{mol}^{-1}$, Weiss 常数 $\Theta$ $=-7.29 \mathrm{~K}$, Weiss 常数 $\Theta$ 为负值表明相邻的锰离子之间 存在反铁磁性相互作用. 在 $300 \mathrm{~K}$ 时根据公式 $\mu_{\mathrm{eff}}=$ $\left(8 \chi_{\mathrm{m}} T\right)^{1 / 2}$ 算得的化合物 1 每个 $\mathrm{Mn}$ 的有效磁矩为 6.18 $\mu_{\mathrm{B}}$, 这稍高于根据 $\mathrm{Mn}^{2+}$ 在高自旋时的理论值 $5.92 \mu_{\mathrm{B}}$, 这可能与相邻 $\mathrm{Mn}^{2+}$ 之间的相互作用有关.

如图 8(b)所示, 化合物 2 的摩尔磁化率随着温 度的变化曲线图, 在 $12 \sim 300 \mathrm{~K}$ 范围内的磁化率按 照Curie-Weiss定律 $\left[\chi_{M}=C /(T-\Theta)\right]$ 进行拟合，得到的 结果是: Curie常数 $C_{\mathrm{m}}=4.13 \mathrm{~cm}^{3} \cdot \mathrm{K} \cdot \mathrm{mol}^{-1}$, Weiss 常数 $\Theta$ $=-52.90 \mathrm{~K}$. Weiss常数 $\theta_{c}$ 为负值表明相邻的钴离子之 间存在反铁磁性相互作用. 在 $300 \mathrm{~K}$ 时根据公式 $\mu_{\mathrm{eff}}=$ $\left(8 \chi_{\mathrm{m}} T\right)^{1 / 2}$ 算得的化合物 2 每个 $\mathrm{Co}^{2+}$ 的有效磁矩为 5.30 $\mu_{\mathrm{B}}$, 这稍低于根据 $\mathrm{Co}^{2+}$ 在高自旋时的理论值 $5.78 \mu_{\mathrm{B}}$. 

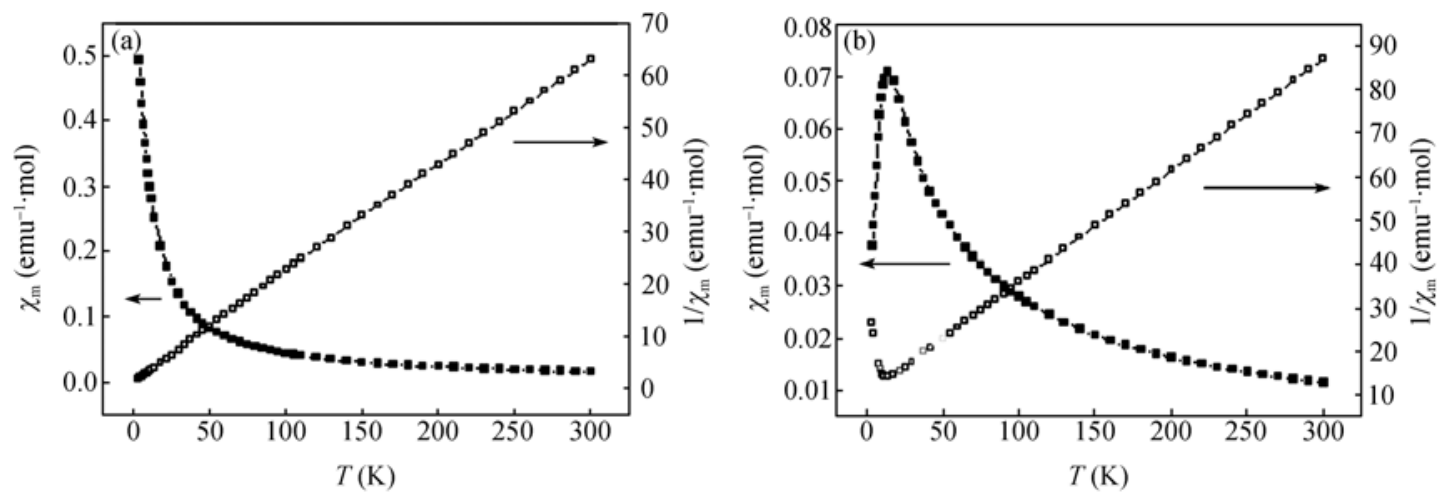

图 8 化合物 1(a)、化合物 2(b)的 $\chi_{\mathrm{m}}$ vs. $T$ 和 1/ $\chi_{\mathrm{m}}$ vs. $T$ 谱图

\section{4 结论}

我们以 8-差基喹啉-5-磺酸和过渡金属离子为金 属中心，通过溶剂热合成方法得到了两个新的三维 的有机-无机配位聚合物. 化合物 1 是 $(6,3)$ 连接的金 红石拓扑网络结构, Schläfli符号为 $\left(4 \cdot 6^{2}\right)_{2}\left(4^{2} \cdot 6^{10} \cdot 8^{3}\right)$.
化合物 2 是通过氢键和 $\pi-\pi$ 相互作用构筑的三维的超 分子骨架结构. 这两个化合物表现出了较强的苂光 发射和反铁磁相互作用，通过研究表明，在相同的有 机配体条件下，改变反应参数可以得到不同结构的 化物. 以 8-羟基喹啉-5-磺酸为配体的其他有机-无机 聚合物的研究还在进行中.

致谢＼cjkstart本工作得到国家重点基础研究发展计划(编号：2006CB806100)、国家自然科学基金杰出青年基金(批 准号：20625102)和国家自然科学基金(批准号：20831D02, 20571030, 20531030, 20371020)资助，特此 一并致谢.

\section{参考文献}

1 Alaerts L, Seguin E, Poelman H, Thibault-Starzyk F, Jacobs P A, D Vos D E. Probing the lewis acidity and catalytic activity of the metal-organic framework $\left[\mathrm{Cu}_{3}(\mathrm{btc})_{2}\right]$ (BTC=Benzene-1,3,5-tricarboxylate). Chem Eur J, 2006, 12: 7353-7363 [DOI]

2 Barbour L J. Crystal porosity and the burden of proof. Chem Commm, 2006, 1163-1168

3 Brunet E, Alhendawi H M H, Cerro C, Mata M J d l, Juanes O, Rodriguez-Ubis J C. Hydrogen storage in a highly porous solid derived from $\gamma$-zirconium phosphate. Angew Chem Int Ed, 2006, 45: 6918-6920[DOI]

4 Chen B L, Ma S Q, Hurtado E J, Lobkovsky E B, Zhou H C. A triply interpenetrated microporous metal-organic framework for selective sorption of gas molecules. Inorg Chem, 2007, 46: 8490-8492 [DOI]

5 Company A, Gómez L, Fontrodona X, Ribas X, Costas M. A novel platform for modeling oxidative catalysis in non-heme iron oxygenases with unprecedented efficiency. Chem Eur J, 2008, 14: 5727-5731 [DOI]

6 Dietzel P D C, Panella B, Hirscher M, Blom R, Fjellvag H. Hydrogen adsorption in a nickel based coordination polymer with open metal sites in the cylindrical cavities of the desolvated framework. Chem Commm, 2006, 959-961

7 Wells A F. Three-Dimensional Nets and Polyhedra. New York: Wiley, 1977

8 Wells A F. Further Studies of Three-Dimensional Nets. American Crystallographic Association. New York (distributed by Polycrystal Book Service, Pittsburgh, PA), 1979

9 Blake A J, Champness N R, Hubberstey P, Li W S, Withersby M A, SchrVder M. Inorganic crystal engineering using self-assembly of tailored building-blocks. Coord Chem Rev, 1999, 183: 117-138[DOI]

10 Fang Q R, Zhu G S, Jin Z, Xue M, Wei X, Wang D J, Qiu S L. A multifunctional metal-organic open framework with a bcu to pology 
constructed from undecanuclear clusters. Angew Chem Int Ed, 2006, 45: 6126-6130[DOI]

11 Xue M, Zhu G S, Li Y X, Zhao X J, Jin Z, Kang E H, Qiu S L. Structure, hydrogen storage, and luminescence properties of three 3D metal-organic frameworks with $\mathrm{NbO}$ and PtS topologies. Cryst Growth \& Des, 2008, 8: 2478-2483[DOI]

12 Xue M, Zhu G S, Ding H, Wu L, Zhao X J, Jin Z, Qiu S L. Six three-dimensional metal-organic frameworks with $(3,4)-,(4,5)-$, and (3,4,5)-connected nets based on mixed ligands: synthesis, structures, and adsorption properties. Cryst Growth \& Des, 2009, 9: $1481-1488 \underline{[\mathrm{DOI}]}$

13 Chen B, Eddaoudi M, Reineke T M, Kampf J W, O’ Keeffe M, Yaghi O M. $\mathrm{Cu}_{2}(\mathrm{ATC}) \cdot 6 \mathrm{H}_{2} \mathrm{O}$ : Design of open metal sites in porous metal-organic crystals (ATC: 1,3,5,7-adamantane tetracarboxylate). J Am Chem Soc, 2000, 122: 11559—11560[DOI]

14 Hoskins B F, Robson R. Design and construction of a new class of scaffolding-like materials comprising infinite polymeric frameworks of 3D-linked molecular rods. A reappraisal of the zinc cyanide and cadmium cyanide structures and the synthesis and structure of the diamond-related frameworks $\left[\mathrm{N}\left(\mathrm{CH}_{3}\right)_{4}\right]\left[\mathrm{Cu}^{\mathrm{I}} \mathrm{Zn}^{\mathrm{II}}(\mathrm{CN})_{4}\right]$ and $\mathrm{Cu}^{\mathrm{I}}\left[4,4^{\prime}, 4^{\prime \prime}, 4^{\prime \prime \prime}\right.$-tetracyanotetraphenylmethane $] \mathrm{BF}_{4} \cdot \mathrm{XC}_{6} \mathrm{H}_{5} \mathrm{NO}_{2} \cdot \mathrm{J} \mathrm{Am}$ Chem Soc, 1990, 112: 1546-1554

15 Sun J Y, Weng L H, Zhou Y M, Chen J X, Chen Z X, Liu Z C, Zhao D Y. QMOF-1 and QMOF-2: Three-dimensional metal-organic open frameworks with a quartzlike topology. Angew Chem Int Ed, 2002, 41: 4471-4473 [DOI]

16 Eddaoudi M, Kim J, O'Keeffe M, Yaghi O M, $\mathrm{Cu}_{2}\left[o-\mathrm{Br}-\mathrm{C}_{6} \mathrm{H}_{3}\left(\mathrm{CO}_{2}\right)_{2}\right]_{2}\left(\mathrm{H}_{2} \mathrm{O}\right)_{2} \cdot(\mathrm{DMF})_{8}\left(\mathrm{H}_{2} \mathrm{O}\right)_{2}$ : A framework deliberately designed to have the NbO structure type. J Am Chem Soc, 2002, 124: 376-377 [DOI]

17 Rosi N L, Eddaoudi M, Kim J, O’Keeffe M, Yaghi O M. Infinite secondary building units and forbidden catenation in metal-organic frameworks. Angew Chem, 2002, 114: 294-297[DOI]

18 Gable R W, Houskins B F, Robson R J. Synthesis and structure of $\left[\mathrm{NMe}_{4}\right]\left[\mathrm{CuPt}(\mathrm{CN})_{4}\right]$ : An infinite three-dimensional framework related to PtS which generates intersecting hexagonal channels of large cross section. Chem Soc Chem Commun, 1990 762-763

19 Gunderman B J, Squattrito P J. Structural variations in layered alkali metal naphthalenesulfonates. Inorg Chem, 1995, 34: 2399$2406[$ DOI]

20 Dalrymple S A, Shimizu G K H. Anion exchange in the channels of a robust alkaline earth sulfonate coordination network. Chem Eur J, 2002, 8: 3010-3015 $\underline{\text { [DOI] }}$

21 Wu H, Dong X W, Liu H Y, Ma J F, Li S L, Yang J, Liu Y Y, Su Z M. Influence of anionic sulfonate-containing co-ligands on the solid structures of silver complexes supported by 4,4'-bipyridine bridges. Dalton Trans, 2008, 5331-5341

22 Rao H Y, Taob J, Ng S W. Polymeric diaqua-8-hydroxyquinolinyl-5-sulfonatozinc(II). Acta Cryst, 2003, E59: m859-m860

23 Wang Y, Wang L, Xu J N, Zhu G S. Poly[diaqua $\left(\mu_{3}-8\right.$-oxidoquinoline-5-sulfonato- $\left.\kappa^{4} \mathrm{~N}, \mathrm{O}^{8}: \mathrm{O}^{8}: \mathrm{O}^{5}\right)$ nickel(II)]. Acta Cryst E, 2009, E65: $\mathrm{m} 902-\mathrm{m} 902[\mathrm{DOI}]$

24 Sheldrick G. M. SADABS, Program for Empirical Absorption Correction for Area Detector Data. Göttingen: University of Göttingen, 1996

25 Sheldrick G M. SHELXS 97, Program for Crystal Structure Refinement. University of Göttingen: Göttingen, 1997

26 Xue M, Zhu G S, Fang Q R, Guo X D, Qiu S L. Solvothermal synthesis, structure and magnetism of two novel 3D metal-organic frameworks based on infinite helical Mn - O - C rod-shaped building units. J Mol Struct, 2006, 796: 165-171 [DOI]

27 Fabelo O, Pasán J, Lloret F, Julve M, Ruiz-Pérez C. 1,2,4,5-benzenetetracarboxylate- and 2,2'-bipyrimidine-containing cobalt(II) coordination polymers: Preparation, crystal structure, and magnetic properties. Inorg Chem, 2008, 47: 3568 - 3576 [DOI]

28 Halls M D, Tripp C P, Schlegel H B. Structure and infrared (IR) assignments for the OLED material: $N, N^{\prime}$-diphenyl- $N, N^{\prime}$ bis(1-naphthyl)-1, 1'-biphenyl-4, 4"-diamine (NPB). Phys Chem Chem Phys, 2001, 3: 2131-2136[DOI]

29 Chen W, Peng Q, Li Y D. Luminescent bis-(8-hydroxyquinoline) cadmium complex nanorods. Cryst Growth \& Des, 2008, 8: $564-567 \underline{\text { [DOI] }}$ 


\title{
Synthesis, crystal structure and properties of inorganic-organic hybrid polymers based on 8-hydroxylquinoline-5-sulfonic acid
}

\author{
WANG Ying, XUE Ming, XU JiaNing, ZHU GuangShan \& QIU ShiLun* \\ State Key Laboratory of Inorganic Synthesis and Preparative Chemistry, Jilin University, Changchun, 130012, China
}

\begin{abstract}
Two new inorganic-organic hybrid polymers, $\mathrm{Mn}(\mathrm{QS})\left(\mathrm{H}_{2} \mathrm{O}\right)$ (1) and $\mathrm{Co}(\mathrm{QS})\left(\mathrm{H}_{2} \mathrm{O}\right)_{2}$ (2) $\left(\mathrm{H}_{2} \mathrm{QS}=8\right.$-hydroxylquinoline-5-sulfonic acid), based on 8-hydroxylquinoline-5-sulfonate ligand, have been synthesized under solvothermal conditions and their structures were solved by single-crystal X-ray diffraction analysis. Compound $\mathbf{1}$ is a three-dimensional open framework with rutile topology structure, and compound $\mathbf{2}$ is a three-dimensional supramolecular structure. These compounds were characterized by powder XRD, infrared spectroscopy, thermogravimetric analysis, fluorescence properties and magnetism properties.
\end{abstract}

Keywords: Inorganic-organic hybrid polymers, 8-hydroxylquinoline-5-sulfonate, Fluorescence properties, Magnetism properties 